\title{
Ophthalmology Practice during Peak of Coronavirus Disease 2019 (COVID-19) Pandemic: A Global Community Perspective
}

\author{
John Zahour, BS ${ }^{1}$ Amin Karadaghy, BS ${ }^{1} \quad$ Eduardo B. Rodrigues, MD ${ }^{1} \quad$ Oscar A. Cruz, MD ${ }^{1}$ \\ ${ }^{1}$ Department of Ophthalmology, Saint Louis University School of \\ Medicine, Saint Louis, Missouri \\ J Acad Ophthalmol 2020;12:e159-e164.

\begin{abstract}
Address for correspondence Eduardo B. Rodrigues, MD, Department of Ophthalmology, Saint Louis University School of Medicine, 1755 South Grand Boulevard, Saint Louis, MO 63104
\end{abstract} \\ (e-mail: eduardo.rodrigues@health.slu.edu).
}

\begin{abstract}
Objective The rise of coronavirus disease 2019 (COVID-19) from Wuhan, China, in December 2019 has spread to more than 188 countries and has affected more than 13 million people. In response to this crisis, recommendations by the World Health Organization have changed the practice of current medicine, but there is little research as to how high-volume ambulatory specialties such as ophthalmology are adapting. The purpose of this study is to determine how ophthalmology practices are reacting and changing during the COVID-19 pandemic.

Methods Approval was obtained from Saint Louis University School of Medicine Institutional Review Board. An anonymous survey was made on Google Forms and distributed to ophthalmologists throughout the world. Questions were divided into five sections: demographics, general questions, inpatient care/consults, practice management, and personal impact. The survey was opened on March 31, 2020, and closed on April 12, 2020. Statistical analysis was performed using Microsoft Excel.

Results A total of 494 responders from 42 countries completed the survey. Respondents were predominantly practicing ophthalmologists (85\%), with the next highest demographic being ophthalmology trainees (8\%). Fear of spreading COVID-19 to patients or loved ones was the highest source of anxiety among practitioners across all practice settings and continental location $(p=0.003)$. The second source of anxiety varied, with private practitioners identifying financial difficulty compared with employed clinicians listing self-contamination. Anxiety levels were the same throughout all practice settings, ages, and subspecialties $(p=0.2527)$. Ophthalmologists listed ophthalmological Web sites/societies, discussion with colleagues, and social media as primary sources of guidance with no difference based on practice setting $(p=0.143)$. Finally, all continents increased their application of telemedicine as a patient care

Keywords

- survey

- COVID

- coronavirus

- guidelines

- telemedicine modality, with North America expanding significantly more than other continents. Conclusion The COVID-19 pandemic has drastically changed ophthalmology practice and has added a high level of stress to ophthalmologists globally. These results demonstrate that clinicians are largely alike across age group, country, and specialty, but key differences in source of anxiety and in application of telemedicine highlight diversity in culture and reaction to the pandemic.
\end{abstract}

received

May 6, 2020

accepted

July 30,2020
DOI https://doi.org/

10.1055/s-0040-1716518. ISSN 2475-4757.
Copyright $\odot 2020$ by Thieme Medical Publishers, Inc., 333 Seventh Avenue, New York, NY 10001, USA. Tel: +1(212) 760-0888.
License terms

()(1) $\Theta \circledast$ 
The rise of coronavirus disease 2019 (COVID-19) from Wuhan, China, in December 2019 has spread to more than 188 countries and has affected more than 13 million people. More than 570,000 individuals have died from COVID-19 as of July 13 , $2020 .^{1,2}$ State and Federal authorities have enlisted a variety of guidelines to the public: social distancing, working from home, safe hand hygiene, curfews, and global travel restrictions to mitigate spread of the virus. ${ }^{3}$ Health care systems throughout the world have followed several recommendations that have changed the current practice of medicine, including personal protective equipment (PPE) such as contact gowns, N95 masks, and face shields. ${ }^{2}$ However, there is little research as to how high-volume ambulatory specialties such as ophthalmology, which is uniquely affected by COVID-19, are adapting, and there is little research seeking to obtain a global perspective. The purpose of this study is to conduct an online survey to determine how ophthalmology practices are reacting to and changing during the COVID-19 pandemic on a global scale.

\section{Methods}

We obtained research approval through the Institutional Review Board (IRB) at Saint Louis University School of Medicine (Protocol \#31142). An anonymous research survey containing 22 questions was created on March 31,2020. Of the 21 questions, we made 15 multiple choice questions, 4 check all that apply, and 2 free responses (-Supplementary Appendix A1, available in the online version). The survey was divided into five sections: demographics, general questions, inpatient care/consults, practice management, and personal impact. Each section had three to six questions and all were optional apart from four demographic questions. The survey was written in English.

The survey was opened on March 31, 2020, when transmissions and deaths from COVID-19 had yet to rise to peak severity throughout the world. No money was spent on advertising. A grassroot approach tailored specifically to those in the field of ophthalmology was used to gain participants (-Supplementary Appendix A2, available in the online version). There were three means of distribution: social media, ophthalmology societies, and individual recruitment.

On social media, practicing ophthalmologists or ophthalmology-related pages on Instagram, Facebook, and Twitter were individually messaged with a prewritten invitation to the survey (-Supplementary Appendix A3, available in the online version). Potential survey recipients were initially identified by searching for relevant terms including "Ophthalmology" and "Ophtho." From those pages, additional recipients were identified through suggestions of each social media platform's algorithm. More than 25 ophthalmological societies were contacted by e-mail with the request to help disperse our survey through their listserves (-Supplementary Appendix A4, available in the online version). If a society did not respond to e-mail, their office was called directly to leave a message. Individual recruitment was conducted through WhatsApp and e-mail. A prewritten message was sent to potential responders, and each participant was asked to share the survey with colleagues.

Data analysis was performed using Microsoft Excel, and we analyzed the relationships between location, anxiety levels, sources of stress, age, ophthalmological guidance, and application of telemedicine. Five responses from nonophthalmology-related fields or from industry representatives were excluded from analysis. Statistical testing was performed using Pearson's chi-square test for categorical data and one-way analysis of variance with Turkey HSD ("honestly significant difference" or "honest significant difference") for numerical data, with an $\alpha$-value of 0.05 for both analyses.

\section{Results}

\section{Demographics}

As of April 12, 2020, 494 responders from six continents and 42 countries completed the survey (-Table 1 ). Notably, the largest groups of participants were from South America and North America, tallying to $79.5 \%$ of responders. Less responses were from Asia, Europe, Africa, and Australia. Ages of the cohort ranged from 20 to more than 60 years. More than one-third of participants were 30 to 40 years old, but there was also large representation from the 40 to 50 and 50 to 60 age groups. Practice setting was largely private practice, with lower contributions from academic, hospitalemployed, and Veteran Affairs (VA)/Government/Military. A large majority of responders were practicing ophthalmologists, with the next two largest demographics being residents/fellows and nurses/technicians. Specialties were comprised of surgical retina, comprehensive, medical retina, cataract, cornea, glaucoma, oculoplastic, pediatrics, neuroophthalmology, and oncology.

\section{Geographic Differences in Anxiety Levels and Telemedicine Application}

We compared the location of each participant to average anxiety level, anxiety source, and the application of telemedicine. Anxiety levels were rated on a scale from 0 to $5(0=$ no more anxiety than usual; $5=$ very high anxiety). Overall, stress levels facing clinicians were high (mean \pm standard deviation: $3.6 \pm 1.3$ ) and varied between continents, with significantly higher numbers in North America and South America than in Europe and Asia $(p=0.0020)$. Sources of anxiety included the fear of spreading COVID-19 to patients or loved ones, self-contamination, risk of financial difficulty, potentially poor malpractice coverage, medical supply insecurity, and food insecurity at home. Across North and South America, Europe, and Asia, the number one cause of concern was spreading the virus to patients or loved ones $(p=0.003)$. The second most common cause of concern in North America, South America, and Europe was financial difficulty, whereas in Asia it was the fear of self-contamination (-Fig. 1A).

We also collected information about the adoption of telemedicine before and during the pandemic. Of the responders, $82 \%$ across the globe did not have access to 
Table 1 Demographics of participants $(N=498)$

\begin{tabular}{|c|c|}
\hline Continental location & Percentage of responders \\
\hline South America & 50.3 \\
\hline North America & 29.2 \\
\hline Asia & 9.9 \\
\hline Europe & 9.2 \\
\hline Africa & 1.0 \\
\hline Australia & 0.4 \\
\hline Age group (years) & Percentage of responders \\
\hline $20-30$ & 7.0 \\
\hline $30-40$ & 36.0 \\
\hline $40-50$ & 31.7 \\
\hline $50-60$ & 16.8 \\
\hline $60+$ & 8.6 \\
\hline Practice setting & Percentage of responders \\
\hline Private practice & 69.7 \\
\hline Academia & 18.2 \\
\hline Hospital employment & 10.0 \\
\hline Veteran affairs & 2.0 \\
\hline Participant title & Percentage of responders \\
\hline Practicing ophthalmologist & 86.1 \\
\hline $\begin{array}{l}\text { Ophthalmology trainee } \\
\text { (resident/fellow) }\end{array}$ & 8.4 \\
\hline Nurse/technician & 2.9 \\
\hline $\begin{array}{l}\text { Administrator/ } \\
\text { practice manager }\end{array}$ & 1.4 \\
\hline Optometrist & 1.0 \\
\hline Nurse practitioner & 0.2 \\
\hline Practice specialty & Percentage of responders \\
\hline Surgical retina & 31.7 \\
\hline Comprehensive & 17 \\
\hline Medical retina/uveitis & 15.1 \\
\hline Cataract & 8.8 \\
\hline Cornea/refractive & 7.2 \\
\hline Glaucoma & 5.1 \\
\hline Pediatrics & 3.1 \\
\hline Oculoplastics & 2.9 \\
\hline Neuro-ophthalmology & 1.2 \\
\hline Oncology & 0.2 \\
\hline Unspecified & 7.8 \\
\hline
\end{tabular}

telemedicine before the pandemic, but $41 \%$ were now implementing it and $26 \%$ planned to practice it soon. For active or suspected COVID-19 infections, 35\% of participants used remote telehealth during consultations. Internationally, all continents increased their application of telemedicine as a patient care modality, but at varied rates. North America was noted to have the highest increase in usage (5.8-fold increase), whereas South America, Europe, and Asia had lower rates of change (-Fig. 1B).

\section{Practice-Specific Differences in Anxiety and Ophthalmological Guidance}

Across all health care settings, the chief concern of responders was once again spreading COVID-19 to a patient or loved one $(p=0.005)$. Self-contamination was the second highest cause of distress in physicians working in academia, hospitals, and VA. In contrast, for physicians working in private practice, financial difficulty was the next highest source of stress (-Fig. 1C). When stratified by ophthalmological specialty, there was no statistical difference in anxiety level $(p=0.2527)$. As for specialty-specific guidance, the majority of participants chose between ophthalmological societies/ Web sites, discussion with colleagues, local institutions, social media, and the Centers for Disease Control and Prevention (CDC) as their primary means of clinical direction. Globally, many physicians were satisfied with the amount of advice provided to them (mean: 7/10 satisfaction level) and believed that the responses by health care systems were appropriate.

In general, the most common sources of ophthalmology guidance came from ophthalmological Web sites/societies (56\%), discussion with colleagues (23\%), and social media $(13 \%)$, and this did not significantly vary by practice setting $(p=0.143)$. As per recommendations, clinic and surgical numbers decreased during the pandemic, as more than $45 \%$ of ophthalmologists saw less than $25 \%$ of their usual surgical volume. It was also noted that top reasons ophthalmologists continued to see patients in the outpatient setting were early postoperative checkups (74\%), anti-vascular endothelial growth factor (VEGF) injections (54\%), and intraocular pressure checks (32\%). Other precautions enforced by different settings and individual practices included reduced hours in the clinic, minimizing staff, increased PPE, transparent shields at front desk, hand disinfection at the front desk, breath shields on slit lamps, screening physicians for fever, screening patients with questionnaires, and application of telemedicine.

\section{Limited Variation in Anxiety by Age Group}

Lastly, we compared the age group of each responder to average anxiety level, root of stress, and source of ophthalmological recommendations. Average stress levels ranged from 3.1 to 3.7, and there was no statistical difference between age groups ( $p=0.1116)$. Moreover, sources of stress did not significantly differ between age groups $(p=0.619)$. Finally, direction from ophthalmological guidance did not differ significantly by age $(p=0.471)$.

\section{Discussion}

This study aimed to inspect the psychological status and response of those in the field of ophthalmology throughout the global community to the COVID-19 pandemic. Internationally, it is important to note that the response of clinicians throughout the world is complicated and multifactorial. For example, when COVID-19 first spread to the United States, there was a shortage of viral testing kits; therefore, the lack of detectability was a major concern to both society and individuals. For this reason, we suspect that higher stress levels in North America may roughly correlate with their 


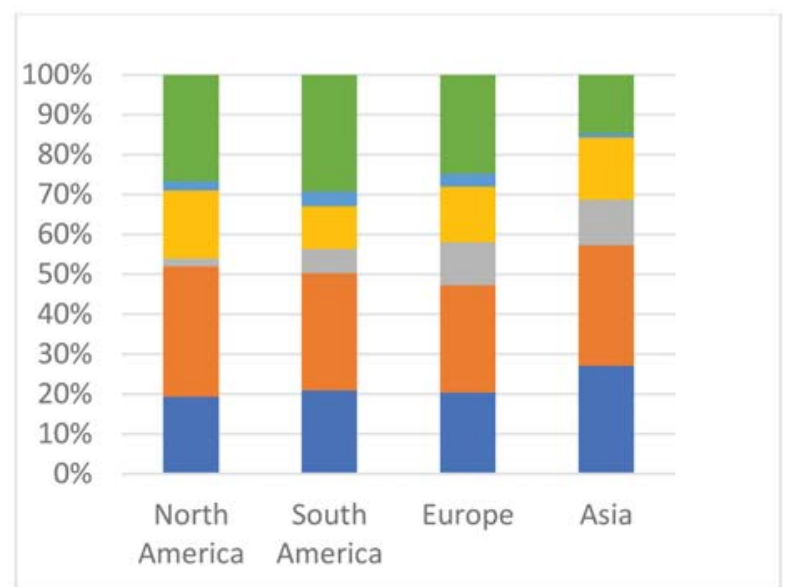

A

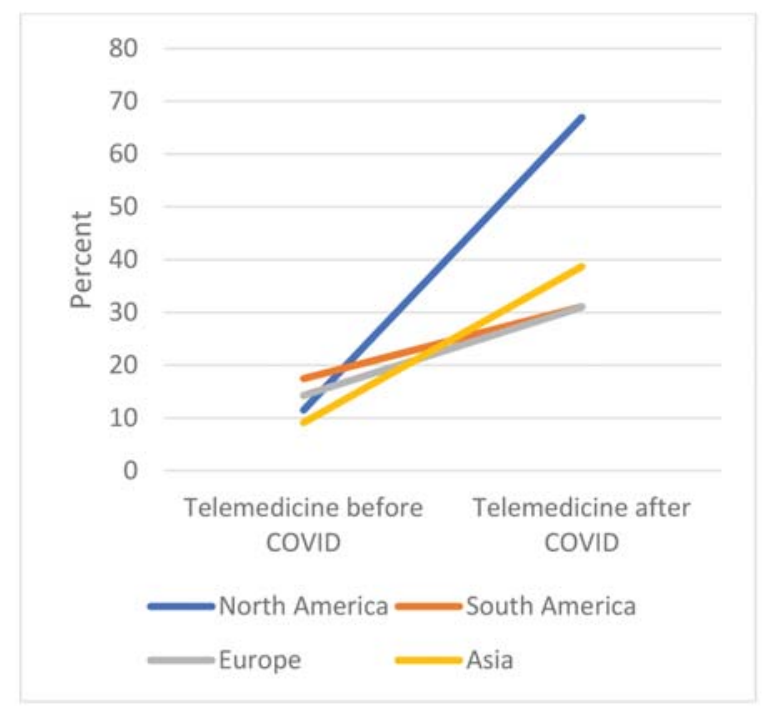

B

Fig. 1 (A) Breakdown of anxiety source by continent using the chi-square test ( $p=0.003$ ). Green, financial difficulty; light blue, supply insecurity at home; yellow, medical supply insecurity; gray, poor malpractice coverage; orange, fear of spreading virus to loved ones; dark blue, fear of self-contamination. (B) Expansion of telemedicine before and after COVID-19 using the chi-square test ( $p=0.003)$. Blue, North America; orange, South America; gray, Europe; yellow, Asia. (C) Practice setting versus anxiety source $(p=0.005)$. Green, financial difficulty; light blue, supply insecurity at home; yellow, medical supply insecurity; gray, poor malpractice coverage; orange, fear of spreading virus to loved ones; dark blue, fear of self-contamination.

number of confirmed cases. During the 12 days this survey was open, North America began to emerge as the next COVID-19 epicenter, and, to date, the highest number of confirmed COVID-19 cases is currently in the United States, with more than 3.3 million. $^{1}$ On the other hand, in countries like China, nosocomial spread of infection was a major issue and likely contributed to rapid spread of the virus. ${ }^{4}$ Therefore, the Chinese health care system has implemented a "Make No Mistake" policy where ophthalmologists were required to wear full PPE for all patients to prevent spread, which is not used in other countries. ${ }^{3}$ Whatever the reasons, only time will tell how different regions will continue to react as the pandemic progresses.

We also observed that physicians continued to practice the pillars of beneficence and nonmaleficence. Across every continent and every practice setting, the clinician's top priority

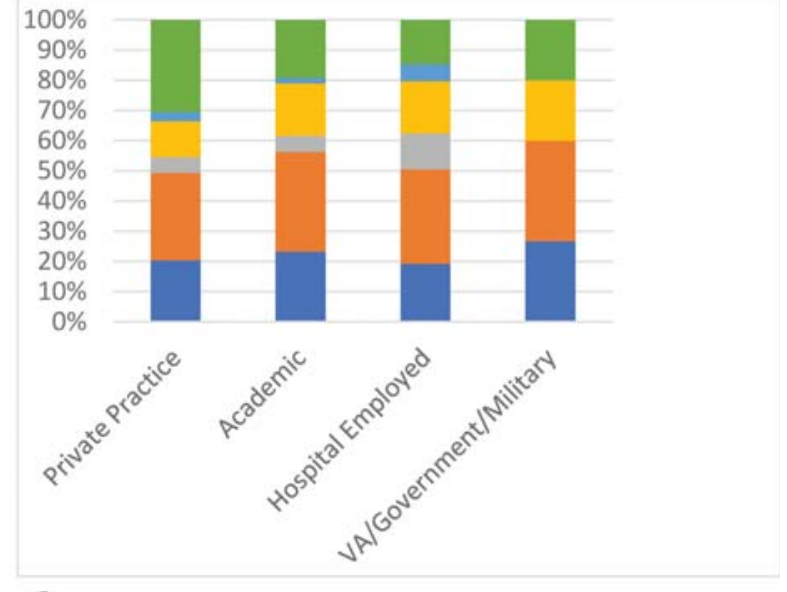

C was preventing the spread of the virus to patients and loved ones. Without necessary intervention, 8 out of every 10 individuals worldwide would be affected, accounting for at least 2.2 million deaths in the United States alone. ${ }^{5}$ After that, the second most common cause of anxiety appeared to reflect more individualized values. By practice setting, we found that the second most common cause of anxiety for private practitioners was financial instability, whereas every other practice setting listed self-contamination as their next top cause of concern. When partitioned by continent, we discovered that while the second most common source of concern for most continents was financial difficulty, Asia was the only continent whose second source was self-contamination. We propose that this is likely explained by the initial spread of COVID-19 out of Wuhan, China. With this information closer to home, we felt that this could be a potential explanation. 
Our data surmised that responders turned toward evidencebased medicine for guidance. In these uncertain times, it is crucial to stay closely attuned to evolving clinical guidance as we continue to learn more about COVID-19. ${ }^{4}$ Furthermore, practice volumes were universally decreased in accordance with guidelines from the American College of Surgeons, ${ }^{6}$ and a vast majority of clinicians implemented two or more of the recommendations from the American Academy of Ophthalmology when a patient needed to be seen. ${ }^{7}$ Whether by ophthalmological societies, word of mouth, social media, or the CDC, clinicians in ophthalmology listened to recommendations.

Other surveys that gauged physician responses to the pandemic had comparable results to our analysis. Although the types of questions varied between each survey and its respective specialty, the authors found that general clinical practices were similar. For example, one survey distributed to more than 1,000 U.S. and international participants by the American Society of Retina Specialists (ASRS) from March 23 to March 29, 2020, found practitioners were also decreasing their clinic volume. In fact, $40 \%$ of U.S. responders and $44 \%$ of international responders reported their patient volume as less than $25 \%$ its usual capacity. ${ }^{8}$ This nicely parallels our data, which demonstrated that more than $45 \%$ of our responders saw less than $25 \%$ of their normal patient volume.

Furthermore, other fields outside of ophthalmology took similar actions. The authors felt that the field of dentistry would also enlist similar safety precautions to ophthalmology given that practitioners in both specialties find themselves close to patient's ocular and oral membranes, increasing the chance of potential aerosol transmission of the virus. One survey administered by the American Dental Association during the week of March 23 to more than 68,000 U.S. participants provided insight into patient management during the pandemic. Of the 19,000 responders, $76 \%$ of dentistry clinics were closed but seeing emergent patients, and $19 \%$ of clinics were closed regardless of patient status. ${ }^{9}$ Similar to our data, it appeared that dentists across the United States were implementing strict safety precautions, debatably even more so than ophthalmology. Nonetheless, both fields strongly valued patient and practitioner safety, and we extrapolate that this was a common motive among all health care workers during this time of uncertainty.

Finally, and perhaps most notably, our evidence suggested that there has been a drastic boost in the application of telemedicine during the COVID-19 pandemic, with significantly higher rates of expansion in North America than in the rest of the world. Federal authorities in the United States have waived normal privacy guidelines within categories of telemedicine to expedite and broaden this billable practice across the country. ${ }^{3}$ We speculated that this service has allowed physicians to still see their patients while decreasing the economic burden of the pandemic, which could be the logic behind the high expansion rate in North America.

The authors felt that this survey provided an accurate portrayal of the virus's impact in the U.S. and internationally, but this study was not without its limitations. Demographic data reveal that a large proportion of survey respondents were from South America, making up about half of the total responses, whereas the next highest proportion from North America represented about one-third of responses. Similarly, a large percentage of respondents represented surgical retina, making up about one-third of responses. The next highest specialty was comprehensive ophthalmology and only represented $17 \%$ of responses. Though we utilized three methods of participant recruitment to diversify responses as much as possible, the majority of respondents were reached through individual emails that were presumably forwarded through communities of colleagues. This opens the possibility of some bias in the results with regard to location and personal beliefs. That said, the survey ultimately yielded good geographic representation with reasonable results, and therefore the authors felt that the evidence from this survey was still valid.

\section{Conclusion}

Like so many other practices of medicine, this survey reflects the drastic disruption the COVID-19 pandemic has had on the field of ophthalmology throughout the world. We felt that this study would be of special interest to ophthalmologists because of the nature of the ophthalmic examination, which demands extremely close face-to-face proximity for even routine visits and because of the early death of the late Dr Li Wenliang, a Chinese ophthalmologist now hailed as an early whistleblower for the virus. ${ }^{10}$ After review, it is apparent that the reactions and practices across all demographics and countries were more alike than different-a warm reminder of unity in otherwise isolated times. Where there were differences, we learn more about individual values such as Asia's higher concern with self-contamination and private practitioners' higher emphasis on financial well-being. We hope that future surveys will focus on integrating larger cohorts with more representation from continents like Africa and Australia. Nevertheless, physicians will undoubtedly continue to care for their patients one way or another. New developments using smartphones and magnifiers to visualize the retina are already underway. ${ }^{11}$ Therefore, we believe that telemedicine will progress as an instrumental resource to all ophthalmologists, and future research should continue to focus on advancements within this health care modality.

\section{Note}

For the availability of data and material, please contact the corresponding author. Informed consent was obtained from all individual participants included in the survey.

Ethical Approval

IRB approved by Saint Louis University IRB on March 31, 2020 (Protocol \#31142).

Funding

None.

Conflict of Interest

None declared. 
e164 Ophthalmology Practice during Peak of COVID-19 Pandemic Zahour et al.

\section{Acknowledgments}

The authors would like to acknowledge St. Louis Ophthalmological Society, Retinography, Brazilian Retina and Vitreous Society, Eye News, and Latinous for their collaboration and distribution of our survey.

\section{References}

1 Dong E, et al. Coronavirus COVID-19 Global Cases by the Center for Systems Science and Engineering (CSSE) at John Hopkins University. ArcGIS Dashboards 2020. Available at: aregis.com/ apps/opsdashboard/index.html\#/ bda7594740fd40299423467b448e9ecf6

2 She J, Jiang J, Ye L, Hu L, Bai C, Song Y. 2019 novel coronavirus of pneumonia in Wuhan, China: emerging attack and management strategies. Clin Translational Med 2020;9(01):19

3 Olivia Li JP, Shantha J, Wong TY, et al. Preparedness among Ophthalmologists: During and Beyond the COVID-19 Pandemic. Ophthalmology 2020;127(05):569-572

4 Adalja AA, Toner E, Inglesby TV. Priorities for the US Health Community Responding to COVID-19. JAMA 2020;323(14): 1343-1344

5 Ferguson NM, Laydon D, Nedjati-Gilani G, et al. Impact of nonpharmaceutical interventions (NPIs) to reduce COVID19 mortality and healthcare demand. Available at: https://www.imperial.ac. uk/media/imperial-college/medicine/sph/ide/gida-fellowships/ Imperial-College-COVID19-NPI-modelling-16-03-2020.pdf

6 American College of Surgeons. COVID-19: Guidance for Triage of Non-Emergent Surgical Procedures. Available at: https://www. facs.org/covid-19/clinical-guidance/triage. Accessed March 17, 2020

7 American Academy of Ophthalmology. Important Coronavirus Updates for Clinicians. Available at: https://www.aao.org/headline/alert-important-coronavirus-context. Accessed April 15, 2020

8 American Society of Retina Specialists. COVID-19 Survey March 24, 2020. Available at: https://files.constantcontact.com/ 98b7aceb001/ef43bfba-942b-4e1c-b810-35502bbacb07.pdf. Accessed July 10, 2020

9 American Dental Association. COVID-19: Economic Impact on Dental Practices (Summary Results). March 2020. Available at: https://www.ada.org/en/science-research/health-policy-institute/covid-19-dentists-economic-impact. Accessed July 10, 2020

10 American Academy of Ophthalmology. Coronavirus Kills Chinese Whistleblower Ophthalmologist. Available at: www.aao.org/ headline/coronavirus-kills-chinese-whistleblower-ophthalmol. Accessed February 11, 2020

11 Salzman IJ. Thoughts from Your Colleagues. EyeNet Magazine. Available at: https://www.aao.org/eyenet/article/thoughts-fromyour-colleagues-56?august-2020 